\title{
Hydroxychloroquine and chronic renal failure
}

\section{Bekhtaoui.C, Bouguedra.H, Chadou.H, Boudia .F, Derbale.F and Toumi.H*}

University hospital establishment Oran pharmacovigilance service Pharmaceutical development research laboratory, Oran University 1 Algeria.

Corresponding Author: Toumi. H., University hospital establishment Oran pharmacovigilance service Pharmaceutical development research laboratory, Oran University 1 Algeria.

\section{Received date: Augusł 16, 2020; Accepted date: August 24, 2020; Published date: September 08, 2020}

Citation: Bekhtaoui. C., Bouguedra. H., Chadou. H., Boudia F., Derbale.F and Toumi.H, (2020) Hydroxychloroquine and chronic renal failure J, Pharmaceutics and Pharmacology Research 3(1); DOI: 10.31579/2693-7247/012

Copyright: (C) 2020, Toumi.H, This is an open access article distributed under the Creative Commons Attribution License, which permits unrestricted use, distribution, and reproduction in any medium, provided the original work is properly cited.

\section{Introduction}

In Algeria, the essentially hydroxychloroquine therapeutic protocol was adopted since March 23, 2020, to which patients with Covid-19 were subjected. This protocol has proven its "almost total" effectiveness by noting a recovery of $98.2 \%$ out of a total of 16,000 cases treated [1].

Hydroxychloroquine (HCQ) is preferred for its better expected tolerance, based on the publications of preliminary work by several teams that we analyzed previously with a further three months' follow-up.

On the pharmaceutical level, the pharmacology of this molecule is of interest and emphasizes the interest of pharmacokinetic studies to define the optimal dosage regimen for patients with a particular statue (Renal Insufficiency - Hepatic Insufficiency - Fatty) COVID-19. On the basis of this work, dosage adjustments can be proposed and the chronology of administration can be optimized with therapeutic monitoring of the drugs to be performed to personalize the individual dosage regimen.

Therefore, the role of the hospital pharmacist is essential in the pandemic context to optimize and modify practices by developing new prescribing protocols while ensuring that adverse events are well managed.

\section{Case Description}

This is an 18 year old patient with a weight of $65 \mathrm{~kg}$ confirmed COVID19 positive suffering from a malformative nephropathy, admitted on 05/11/2020 to the service COVID university hospital Establishment UHE,

\begin{tabular}{|l|l|l|l|l|l|}
\hline $\begin{array}{l}\text { Duration of } \\
\text { hospitalization }\end{array}$ & D1 & D2 & D3 & D4 & D5 \\
\hline $\begin{array}{l}\text { Clearance ml / min / } \\
\begin{array}{l}\mathbf{1 . 7 3} \mathbf{~ m}^{2} \\
\text { (MDRD) }\end{array}\end{array}$ & & 6.28 & 6.51 & 6.91 & 7.31 \\
\hline $\begin{array}{l}\text { Clearance ml / min } \\
\text { (cockroroft) }\end{array}$ & 9.73 & 9.49 & 10.09 & 10.57 & 11.09 \\
\hline
\end{tabular}
positive. $400 \mathrm{~ms})$. per day for the next 4 days, times daily.

hospitalisation was as follow

ORAN, Algeria after suspicion contamination whose father is confirmed

The patient being asymptomatic with a general preserved state, diuresis $1100 \mathrm{ml}$, an arterial pressure $=120 / 80$, a temperature: $36.2^{\circ} \mathrm{c}$, a heart rate $=90 \mathrm{bpm}$, an oxygen saturation $\mathrm{SAO} 2=98 \%$, and presenting at 1 " ECG a regular sinus rhythm $(\mathrm{PR}=0.16 \mathrm{~s}, \mathrm{QRSfin}$, normal $\mathrm{AXIS}, \mathrm{QTc}=$

The patient was put on specific treatment according to the national protocol. The latter will use the following drugs: Hydroxychloroquine $200 \mathrm{mg}$ at a rate of $200 \mathrm{mg}$ x 3 times per day for 10 days in combination with Azithromycin $250 \mathrm{mg}$ : $500 \mathrm{mg}$ on the first day followed by $250 \mathrm{mg}$

In view of the severe renal impairment (clearance $=8 \mathrm{ml} / \mathrm{min}$ ) it was recommended a dosage adjustment of HCQ following nephrological advice, i.e. a $50 \%$ reduction of the standard dose to 100mg HCQ three

After a request from the Therapeutic Optimisation Unit of the UHE Pharmacovigilance Department, capsules with the recommended dosage were prepared; combined with a pharmaceutical analysis of the patient's medical prescription by the clinical pharmacy team.

The evolution of the patient's clearance during the first five days of

Table I: Evolution of patient clearance during the first five days of hospitalisation

\section{Hydroxychloroquine pharmacokinetics and toxicity} profile

Hydroxychloroquine is an anti-malarial from the class of amino-4quinolines which has found its place in the ministerial protocol proposed by the expert committee in response to the COVID-19 pandemic [2].

This molecule is rapidly absorbed in the digestive tract after oral administration, it has a large volume of distribution and it is strongly linked to plasma proteins with a strong distribution in the following tissues: kidney, liver, lungs, cells containing melanin such as those of the eyes and the skin. It crosses the placenta. It is mainly metabolized in monodesethylchloroquine (cletoquine) and bisdesethylchloroquine in the liver. Monodesethylcholoroquine has been reported to have some activity. Elimination is mainly renal: $50 \%$ in unchanged form and $10 \%$ in monodesethylchloroquine form. The half-life of hydroxychloroquine is approximately 30 days. This molecule therefore accumulates in the 
tissues and can be found several weeks to months in the tissues. Hydroxychloroquine is a substrate for CYP2C8, CYP3A4 and CYP2C6, possible pharmacokinetic drug interactions should be taken into account $[2,3]$.

- Contraindications: retinopathies, hemolytic anemia, porphyria, G6PD deficiency, myasthenia gravis [3].

- The most frequent adverse effects of hydroxychloroquine are: headache, rash, pruritus, gastrointestinal disorders (nausea, vomiting, and diarrhea), visual disturbances and cardiomyopathy. These adverse reactions have been reported to be more frequent and severe at high doses and over the long term.

- Retinal Toxicity: Depends on daily dose $(>400 \mathrm{mg} / \mathrm{J})$, cumulative dose $(>1000 \mathrm{~g})$, patient risk factors and duration of treatment $>5$ years.

- Retinal toxicity is not questioned in this study setting due to the short duration of treatment (10J).

- Cardiac toxicity: the cardiotoxicity of hydroxychloroquine is attributable to an action similar to the action of quinidine which causes: a negative inotropic action, inhibits spontaneous diastolic depolarization, slows conduction, lengthens the effective refractory period and raises the electrical threshold. This results in a depression of contractility, an alteration in conductivity, a decrease in excitability and a possible abnormal stimulus responsible for reentry mechanisms. Cardiac arrest may be the first manifestation of an overdose. The hypokalemia associated with these overdoses is correlated with the severity of intoxication. The mechanism seems to be an intracellular transport of potassium instead of a real potassium deficit.

The serious side effects that hydroxychloroquine can cause, mainly cardiac arrhythmias, are known, in particular the risk of prolonging the QTc interval. And these effects can be further enhanced by the simultaneous administration of other drugs such as azithromycin. In addition, these two active ingredients can damage the liver, kidneys or nervous system and cause a drop in blood sugar. Serious signs of cardiac toxicity have been reported in blood concentrations of hydroxychloroquine ranging from 2.05 to $29.40 \mathrm{mcmol} / \mathrm{l}$. It is recommended to monitor plasma concentrations in patients receiving this treatment for COVID-19 and to provide cardiac monitoring [3, 4].

Whipple's disease, a systemic infectious disease caused by Tropheryma whipplei or the standard treatment, is the combination of doxycycline and hydroxychloroquine. In this indication, the HCQ is administered at the same dosage as in the proposed covid_19 protocol (600 mg per day). The target HCQ plasma levels described in the literature are $1+/-0.2 \mathrm{ug} / \mathrm{ml}$ [5].

\section{Hydroxychloroquine and kidney failure:}

HCQ is mainly excreted via the kidneys. Particular attention paid to patients with renal insufficiency conducive to developing early cardiotoxicity. It was recommended to use lower doses of HCQ [6,7] varying according to the glomerular filtration rate (table 1) and the type of dialysis (table 2) it is described in the literature that dialysis does not significantly lower plasma concentrations. The dose reductions illustrated in the tables are recommended in the context of systemic inflammatory pathologies such as lupus, an indication in which the taking of HCQ is long-term with low dosages $(200 \mathrm{mg})$ and in single dose for objective of avoid cumulative retinal toxicity.

Due to the lack of experience with dosage adjustment and in order to avoid early cardiac toxicity in patients with renal failure; a $50 \%$ reduction in HCQ has been proposed. Cardiac evaluation of this patient showed no abnormalities.

\begin{tabular}{|l|l|}
\hline Renal Replacement Therapy & Daily Dose \\
\hline CAPD & $\begin{array}{l}\text { Non-dialysis. Dose for one GFR }<10 \\
\mathrm{~mL} / \mathrm{min}\end{array}$ \\
\hline HD & $\begin{array}{l}\text { Non-dialysis. Dose for one GFR }<10 \\
\mathrm{~mL} / \mathrm{min}\end{array}$ \\
\hline HDF/highFlux & $\begin{array}{l}\text { Dialysability not known. Dose for one } \\
\text { GFR }<10 \mathrm{~mL} / \mathrm{min}\end{array}$ \\
\hline CAV/VVHD & $\begin{array}{l}\text { Dialysability not known. Dose for one } \\
\text { GFR }=10-30 \mathrm{~mL} / \mathrm{min}\end{array}$ \\
\hline
\end{tabular}

Table II: Recommended daily dose of hydroxychloroquines according to glomerular filtration rate for the prevention of retinal toxicity. (GFR)[6]

\begin{tabular}{|l|l|}
\hline GFR (mL/min) & $\begin{array}{l}\text { Max daily dose of } \\
\text { hydroxychloroquine }\end{array}$ \\
\hline $\mathbf{3 0}-\mathbf{5 0}$ & Maximum $75 \%$ of the dose \\
\hline $\mathbf{1 0}-\mathbf{3 0}$ & $25-50 \%$ of the dose \\
\hline$<\mathbf{1 0}$ & $25-50 \%$ of the dose- use with caution \\
\hline
\end{tabular}

Continuous ambulatory peritoneal dialysis (CAPD), intermittent haemodialysis (HD), Haemodiafiltration (HDF) Continuous arteriovenous / veno venous haemodialysis (CAV / VVHD) Glomerular filtration rate (GFR). 
Therapeutic optimization of hydroxychloroquine

At the pharmacovigilance service for the therapeutic optimization unit, a preparation of hydroxychloroquine dosed at $100 \mathrm{mg}$ was carried out according to the following steps:

\section{I-Calculation of the total mass of the active principle}

Total mass of the active ingredient $=$ unit dosage requested $*$ number of capsules to be prepared

$=100 * 30=3000 \mathrm{mg}$

Knowing that:

The number of capsules to prepare $=$ dosage $*$ duration of treatment $=3 * 10=30$

\section{II-Deconditioning of a specialty}

It is the calculation of the number of tablets to be crushed or capsules to empty of their content:

$\mathrm{N} \mathrm{Cp}=$ unit dosage requested $*$ number of capsules to prepare

$$
\mathrm{N} \mathrm{Cp}=100 * 30 / 200=15 \mathrm{cp}
$$

\section{III-Adjustment of the volume of the diluent to be added}

Depending on the size of the desired capsule, the volume of the diluent to be added can be calculated.

For a capsule $\mathrm{N}^{\circ} 1$ we need $0.5 \mathrm{ml}$ of diluent for each capsule.

For 30 capsules you need $15 \mathrm{ml}$ of diluent.

IV-Trituration with mortar of the active ingredient + diluent V-Preparation of the capsule filler

VI-Filling and ejection of capsules

VII-Pillbox

VIII-Labeling

\section{Pharmaceutical care}

\section{Medical prescription:}

- Hydroxychloroquine Gel 100mg 3 * d for 10 days

- Azithromycin $500 \mathrm{mg}$ the first day then $250 \mathrm{mg}$ for 4 days

- Cefotaxime $1 \mathrm{~g} 3 * \mathrm{~d}$

- Enoxaparin 4000 IU for 10 days

Our team carried out the pharmaceutical analysis, various problems related to the drug were detected:

\section{Drug interaction:}

Combination type not recommended (hydroxychloroquine / azithromycin).Hydroxychloroquine can prolong the QT interval. Theoretically, co-administration with other drugs that may prolong the QT interval (azithromycin) may result in additive effects and an increased risk of ventricular arrhythmias, including torsade de pointes and sudden death. In general, the risk of an individual drug or combination of drugs causing a ventricular arrhythmia associated with QT prolongation is largely unpredictable, but may be increased by certain underlying risk factors, such as congenital long QT syndrome, heart disease and electrolyte disturbances (e.g., hypokalemia, hypomagnesemia). In addition, the extent of drug-induced QT prolongation depends on the drug involved and its dosage.Pharmaceutical intervention:

1. Co-administration of hydroxychloroquine with other drugs that can prolong the QT interval should generally be avoided. Patients treated with hydroxychloroquine should be advised to seek prompt medical attention if they develop symptoms which may indicate the presence of torsades de pointes such as dizziness, fainting, palpitations, irregular heartbeat, shortness of breath or fainting. [9].

2. Calculation of the Tisdale Score to predict the risk of prolongation of the QT interval [10,11]: this score is available online (https://www.mdcalc.com/tisdale-risk-score-qt-

prolongation\#evidence)

The calculated score of the patient in question being equal to 6 is equivalent to a low score: Low risk of prolongation of the QT interval; always consider that a higher risk may develop based on clinical course and drug interactions and pharmacokinetics.

On this basis we recommend:

- The ECG, repeated 8 to 12 hours after the start of QT prolongation therapy to look for signs of QT prolongation, with closer monitoring if observed.

- Correct electrolyte abnormalities and maintain blood potassium> 4.0 $\mathrm{mEq} / \mathrm{L}$ and serum magnesium $>2.0 \mathrm{mg} / \mathrm{d}$.

\section{Non-compliance with standards / contra indication:}

Lovenox 4000 IU / d / d for 10 days [12].Reminder:

The risk factors of patients who are candidates for prophylaxis are well known [13, 14 and 15]: age, acute infection, active cancer, history of venous thromboembolic disease (VTE), prolonged immobilization, chronic heart failure and insufficiency respiratory.

The patient in question has two risk factors (covid-19 infection and hospitalization); which calls for the need for thromboprophylaxis:

-Description of the problem with the drug treatment: the patient being insufficient renal end, it is not recommended to use heparin at low molecular weight because of the increase in the risk of haemorrhage.

$\Rightarrow$ Pharmaceutical intervention: Substitution / Exchange

The patient's clearance being less than $15 \mathrm{ml} / \mathrm{min}(8 \mathrm{ml} / \mathrm{min})$ : a substitution with HNF SC (Calciparin according to the establishment's register) is recommended, the recommended dosage is shown in the table below for the thromboembolic risk:

\begin{tabular}{|l|l|l|}
\hline \multicolumn{3}{|c|}{ Thromboembolic risk } \\
\hline Weight & Low & Student \\
\hline $\mathbf{5 0 - 8 0} \mathbf{~ k g}$ & $5000 \mathrm{U} \mathrm{2} * / \mathrm{d}$ & $5000 \mathrm{U} 3 * / \mathrm{d}$ \\
\hline
\end{tabular}

Table IV: Recommended dosage depending on the thromboembolic risk High risk: in case of: active cancer, history of VTE, recent surgery, known thrombophilia.

That said, our patient with a low thromboembolic risk, we recommend a dosage of 5000U $2 * / \mathrm{d}$.

\section{Monitoring to follow for the HNF recommendation:}

Kalemia, Platelet count before treatment and $2 *$ / week during treatment, Liver balance before treatment, monitoring of anti-Xa or TCA is not recommended when heparin is used for preventive purposes [16].

\section{Overdose / supra-therapeutic dosage:}

Cefotaxime (Claforan) $1 \mathrm{G} 3 * \mathrm{~d}$ for 10 days [17].

$\Rightarrow$ Pharmaceutical intervention: Dosage adjustment

In patients with a clearance of less than $10 \mathrm{ml} / \mathrm{min}$; after a normal starting dose, maintenance doses should be reduced by half compared to the 
normal dose without changing the dose interval. A dosage of $500 \mathrm{mg} / 3$ $* \mathrm{~d}$ is recommended after an initial dose of $1 \mathrm{G} / 3 * \mathrm{~d}$.

\section{Monitoring to follow:}

- Liver balance: hydroxychloroquine is metabolized in the liver, it is necessary to evaluate the liver balance before starting treatment. Liver failure causes an accumulation of hydroxychloroquine in the tissues, which is equivalent to an overdose [2].

- Magnesemia: it is necessary to perform magnesemia coupled with a kalemia for optimal cardiac monitoring [8].

\section{References}

1. Arezki.B, (2020). Chloroquine has proven to be effective with the recovery of $98.2 \%$ of patients. El Watan newspaper.

2. C. Samer, F. Curtin, KR Ing-Lorenzini, P. Vetter; (2020). Chloroquine, hydroxychloroquine and COVID-19: Pharmacological evaluation; Department of Acute Medicine Department of Clinical Pharmacology and Toxicology; Geneva University Hospitals

3. Swiss Society of Clinical Pharmacology and Toxicology. (2005). Basics of drug therapy.

4. KATHY MARQUADT, PHARM D; (2001). treatment of hydroxychloroquine overdose; American journal of emergency medicine volume 19 , number 5 ,

5. Y. Zerbib, MP Guillaumont, G. Touati, P. Duhaut, J. Schmidt; (2015). Early cardiotoxicity of hydroxychloroquine; The Journal of Internal Medicine

6. Ashley C, and Currie A, (2009). EdsUK Renal Pharmacy Group., The Renal Drug Handbook, p362.
7. BethelM, et al. (2016). Hydroxychloroquine in patients with systemic lupusery the matosus with end-stage renal disease. J Investig Med; 64: 908-910.

8. Samer C, Curtin F, Ing-Lorenzini KR, Vetter P. (2020). Chloroquine, hydroxychloroquine and COVID-19: Pharmacological evaluation. COVID HUG Guidelines group. p13

9. https://www.mdcalc.com/tisdale-risk-score-qt prolongation \# evidence

10. https://www.mdcalc.com/tisdale-risk-score-qtprolongation\#evidence

11. James E Tisdale1, Heather A Jaynes; (2013). Development and Validation of a Risk Score to Predict QT Interval Prolongation in Hospitalized Patients; Circ Cardiovasc Qual Outcomes. 2013 Jul; 6 (4): 479-87, doi: 10.1161 / CIRCOUTCOMES.113.000152.

12. RCP cefotaxime, ANSM, 2017

13. Amin AN, Varker H, Princic N, Lin J, Thompson S, Johnston S. (2012). Duration of venous thromboembolism risk across a continuum in medically ill hospitalized patients. J Hosp Med.; 7 (3): 231-8.

14. Samama MM, Cohen AT, Darmon JY, Desjardins L, Eldor A, Janbon C, et al. (1999). A comparison of enoxaparin with placebo for the prevention of venous thromboembolism in acutely ill medical patients. Prophylaxis in Medical Patients with Enoxaparin Study Group. N Engl J Med; 341 (11): 793-800.

15. Kearon C, Akl EA, Ornelas J, Blaivas A, Jimenez D, Bounameaux $H$, et al. (2016). Antithrombotic Therapy for VTE Disease: CHEST Guideline and Expert Panel Report. Chest; 149 (2): 315-52.

16. iKb vascular cariology 8th edition-2018.

17. RCP Céfotaxime ANSM report May 2020. 